\title{
Neuronal avalanches and the cortico-striatal network
}

\author{
Jovana J Belić ${ }^{1,2^{*}}$, Andreas Klauss ${ }^{3,4}$, Dietmar Plenz ${ }^{4}$, Jeanette Hellgren Kotaleski,3 \\ From Twenty First Annual Computational Neuroscience Meeting: CNS*2012 \\ Decatur, GA, USA. 21-26 July 2012
}

Neuronal avalanches are spontaneous activity cascades observed in superficial cortical layers with statistical properties expected from the network operating near a critical point [1]. In such a network, neuronal activity on one active site triggers, on average, similar activity at other site and therefore the overall activity does not increase or die out over time. Neuronal avalanches have been found in vitro [1] and in vivo [2], and display long-term stability, diversity, and fast propagation of local synchrony. They characterize networks that have a maximum dynamic range [3] and might play a central role in information transmission [1] and storage [4]. Their activity is characterized by brief bursts lasting tens of milliseconds, separated by periods of quiescence lasting several seconds and when observed with multi-electrode arrays, the number of electrodes activated is well described by a power law with exponent close to -1.5 [5].

Here we study neuronal avalanches in an open-loop system of cortex and striatum. The striatum is the main input structure of the basal ganglia and plays an important role in motor and cognitive functions. Understanding how the striatum responds to cortical inputs has crucial importance for clarifying the overall functions of the basal ganglia. The projection neurons of the striatum have a high threshold for activation and receive excitatory input from different regions of the cerebral cortex. Although the striatum contains several distinct cell types, 90-95\% are GABAergic medium spiny projection neurons (MSNs). These cells are major targets of cortical inputs, the recurrent connection among them mediate weak feedback inhibition and neighboring MSNs are not likely to share cortical inputs [6]. Fast-spiking interneurons are relatively sparse elements of striatal networks. They

\footnotetext{
* Correspondence: belic@kth.se

'Computational Biology, Royal Institute of Technology (KTH), Stockholm, 106 91, Sweden

Full list of author information is available at the end of the article
}

project extensively to nearby MSNs and provide strong feedforward inhibition and seem to be critical nodes governing striatal output [7].

Preliminary experiments indicate that activity bursts in the striatum do not follow a power law with characteristic exponent of -1.5 . Here we developed an abstract model of the cortico-striatal network that reproduces statistics observed in experimental data. We discuss which kind of connectivity between cortex and striatum, as well as connectivity and strength of inhibition within striatum can lead to results that are in line with experimental data.

\section{Acknowledgements \\ Supported by the Erasmus Mundus Joint Doctoral program EuroSPIN.}

\section{Author details}

'Computational Biology, Royal Institute of Technology (KTH), Stockholm, 106 91, Sweden. ${ }^{2}$ Bernstein Center Freiburg, University of Freiburg, Freiburg, 79104, Germany. ${ }^{3}$ Department of Neuroscience, Karolinska Institutet (KI), Stockholm, 171 77, Sweden. ${ }^{4}$ Section on Critical Brain Dynamics, National Institute of Mental Health (NIH), Bethesda, USA.

\section{Published: 16 July 2012}

\section{References}

1. Beggs J, Plenz D: Neural avalanches in neocortical circuits. The Journal of Neuroscience 2003, 23:11167-11177.

2. Gireesh ED, Plenz D: Neural avalanches organize as nested theta and beta/gamma-oscillations during development of cortical layer 2/3. PNAS 2008, 105:7576-7581.

3. Shew W, Yang H, Petermann T, Roy R, Plenz D: Neural avalanches imply maximum dynamic range in cortical networks at criticality. The Journal of Neuroscience 2009, 29:15595-15600.

4. Beggs J, Plenz D: Neural avalanches are diverse and precise activity patterns that are stable for many hours in cortical slice cultures. The Journal of Neuroscience 2004, 24:5216-5229.

5. Klaus A, Yu S, Plenz D: Statistical analyses support power law distributions found in neuronal avalanches. PLOS ONE 2011, 6(5):e19779.

6. Zheng J, Wilson CJ: Corticostriatalcombinatorics: the implication of corticostriatal axonal arborizations. Journal of Neurophysiology 2002, 87:1007-1017.

7. Berke J: Functional properties of striatal fast-spiking interneurons. Frontiers in Systems Neuroscience 2011, 5:1-7.

\section{Biomed Central}


doi:10.1186/1471-2202-13-S1-P122

Cite this article as: Belić et al: Neuronal avalanches and the cortico-

striatal network. BMC Neuroscience 2012 13(Suppl 1):P122.

Submit your next manuscript to BioMed Central and take full advantage of:

- Convenient online submission

- Thorough peer review

- No space constraints or color figure charges

- Immediate publication on acceptance

- Inclusion in PubMed, CAS, Scopus and Google Scholar

- Research which is freely available for redistribution

Submit your manuscript at 